ARTICLE

https://doi.org/10.1038/s41467-019-13072-x

\title{
Functionalized azetidines via visible light-enabled aza Paternò-Büchi reactions
}

\author{
Marc R. Becker ${ }^{1}$, Alistair D. Richardson ${ }^{1} \&$ Corinna S. Schindler ${ }^{1 \star}$
}

Azetidines are four-membered nitrogen-containing heterocycles that hold great promise in current medicinal chemistry due to their desirable pharmacokinetic effects. However, a lack of efficient synthetic methods to access functionalized azetidines has hampered their incorporation into pharmaceutical lead structures. As a [2+2] cycloaddition reaction between imines and alkenes, the aza Paternò-Büchi reaction arguably represents the most direct approach to functionalized azetidines. Hampered by competing reaction paths accessible upon photochemical excitation of the substrates, the current synthetic utility of these transformations is greatly restricted. We herein report the development of a visible light-enabled aza Paternò-Büchi reaction that surmounts existing limitations and represents a mild solution for the direct formation of functionalized azetidines from imine and alkene containing precursors.

\footnotetext{
${ }^{1}$ Willard Henry Dow Laboratory, Department of Chemistry, University of Michigan, Ann Arbor, MI 48109, USA. *email: corinnas@umich.edu
} 
$\mathrm{N}$ itrogen-containing heterocycles are essential structural components for drug design and are currently incorporated in more than $59 \%$ of all pharmaceuticals approved by the US Food and Drug Administration (FDA) ${ }^{1}$. The majority of these contain five- and six-membered pyrrolidine and pyridine derivatives, regardless of the fact that smaller heterocycles, such as the four-membered azetidines (1), are known to display superior physicochemical properties and increased bioavailability, as well as metabolic stability ${ }^{2-6}$. Despite these desirable characteristics, azetidines remain underutilized in current medicinal chemistry, which is a direct result of a lack of efficient synthetic methods for their construction ${ }^{7-9}$. The most important strategy for the synthesis of saturated nitrogen-containing heterocycles relies on unimolecular cyclization reactions via nucleophilic substitution (4, Fig. 1a $)^{10}$. While this approach results in the efficient formation of three-, five-, and six-membered heterocycles, it often fails to yield the four-membered azetidines (11). In general, fourmembered rings are considered the hardest of all to form ${ }^{11}$. The reason for this lack of reactivity lies in the preferred conformation of the acyclic precursors (Fig. 1b). Specifically, the formation of three-membered rings (9) is favorable as $\mathbf{8 b}$ represents both the preferred as well as reactive conformation for cyclization. In comparison, formation of the four-membered azetidines is hampered as it requires access to conformation $10 \mathrm{~b}$, which is higher in energy due to unfavorable eclipsing interactions ${ }^{12}$. Thus, strategies that proceed upon strain release of azabicyclobutanes (5) as three-membered ring analogs were developed as viable alternatives for the synthesis of azetidines ${ }^{13-18}$. In addition, orthogonal strategies for azetidine synthesis were developed that rely on the reduction of more readily accessible $\beta$-lactam

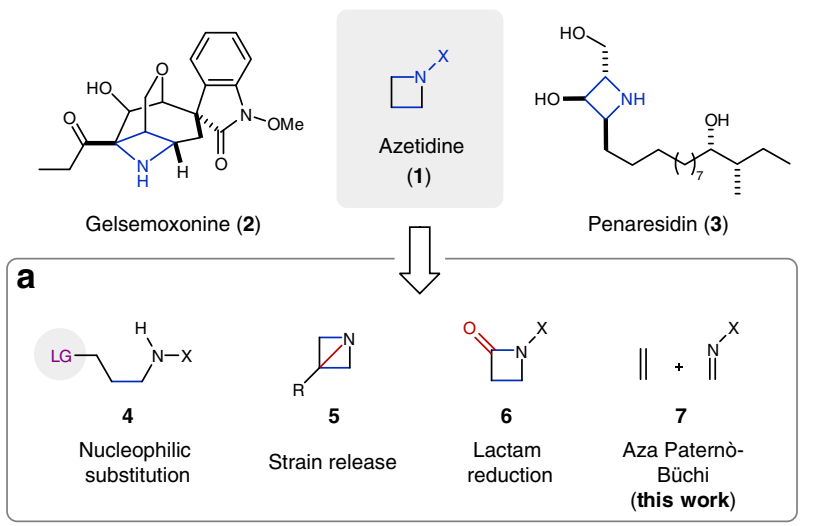

b

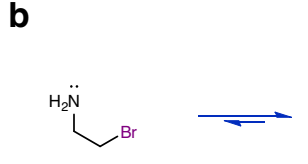

8 a

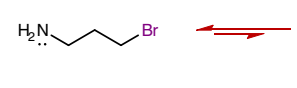

10a

C
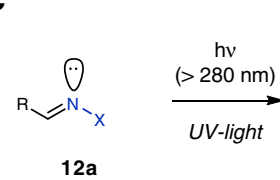
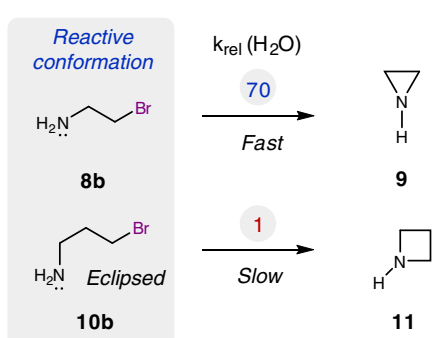

11
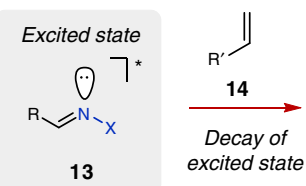

precursors (6) ${ }^{10,19,20}$. Arguably, aza Paternò-Büchi reactions (7) represent the most efficient and direct strategy towards azetidines. Analogous to the Paternò-Büchi reaction ${ }^{21}$, in which an excited state carbonyl undergoes a [2+2] cycloaddition with an alkene, aza Paternò-Büchi reactions rely on imines and alkenes. However, the development of these transformations has met challenges associated with the decreased photoreactivity of imine precursors $^{22,23}$. In particular, the excited state of imines (13) is known to undergo facile and preferential radiationless decay upon rotation about the $\mathrm{C}=\mathrm{N} \pi$-bond $(\mathbf{1 2 b})^{24-27}$. This results in dissipation of electronic energy and a lack of reactivity in [2+2] cycloadditions with alkenes (14) ${ }^{28-42}$ (Fig. 1c). Hence, successful reports of aza Paternò-Büchi reactions are rare and limited to rigid imine- and alkene-containing systems predisposed for cycloaddition in addition to the stringent requirement of high energy ultraviolet (UV) light ${ }^{43-51}$. Consequently, the development of a mild and general reaction protocol for aza Paternò-Büchi reactions relying on visible light ${ }^{52-57}$ would be highly desirable.

Here, we report the development of a visible light-mediated aza Paternò-Büchi reaction, which enables the synthesis of highly functionalized azetidines from readily available imine and alkenecontaining precursors. Notably, this strategy is characterized by its mild conditions, operational simplicity, and scalability. The accessible azetidine products are readily converted into more valuable azetidine building blocks.

\section{Results}

Reaction optimization of aza Paternò-Büchi reaction. At the outset of our studies, we envisioned an orthogonal approach for aza Paternò-Büchi reactions that relies on selective activation of the alkene functionality to avoid excitation of the imine and associated competing reaction paths (Fig. 2). Triplet energies of alkenes $^{58}$ (e.g., styrenes, dienes) are known to be lower than those of functionalized imines ${ }^{59-62}$, and could thus engage selectively in an energy transfer process with a suitable excited photocatalyst to reach the triplet state of the alkene. Subsequent $[2+2]$ cycloaddition with the $\mathrm{C}=\mathrm{N}$ double bond of the imine moiety would then lead to the formation of the desired azetidine product. Importantly, activation of the photocatalyst could be achieved by irradiation with visible light and thus forgo excitation of the imine and associated decay pathways.

Our initial investigations into the development of a mild protocol for an aza Paternò-Büchi reaction centered on oximes such as $\mathbf{1 5}$ due to their facile synthesis and superior stability towards hydrolysis compared to imines. Consistent with literature reports, irradiation of oxime $\mathbf{1 5}$ with UV light resulted in $E / Z$ isomerization of the oxime and only trace amounts of azetidine 16 as the desired aza Paternò-Büchi product ${ }^{63}$ (entry 1, Table 1). Conversion of $\mathbf{1 5}$ with catalytic amounts of xanthone as photosensitizer and UV light following conditions previously reported by Sivaguru and co-workers ${ }^{51}$ resulted in $43 \%$ yield albeit complete conversion of the substrate (entry 2, Table 1). We postulated that substrate decomposition is a direct consequence of the high redox properties of xanthone upon irradiation with UV light ${ }^{64}$. Following our initial hypothesis that selective alkene activation to its corresponding triplet state could lead to a mild reaction protocol for aza Paternò-Büchi reactions, we next evaluated a variety of photocatalysts in combination with lower energy visible light ( $40 \mathrm{~W}$ blue light-emitting diode (LED) light at $427 \mathrm{~nm}$ ). When oxime 15 was irradiated with visible light in the presence of catalytic amounts of $\left[\mathrm{Ru}(\mathrm{bpy})_{3}\right]\left(\mathrm{PF}_{6}\right)_{2}$, no formation of azetidine 16 was observed (entry 3, Table 1). Nevertheless, when $\operatorname{Ir}(\text { ppy })_{3}$ was used as photocatalyst under otherwise identical conditions, the desired aza Paternò-Büchi product 16
Fig. 1 Previous strategies towards azetidines and this approach. a Select synthetic strategies. b Challenges in nucleophilic substitution reactions c Challenges in aza Paternò-Büchi reactions 


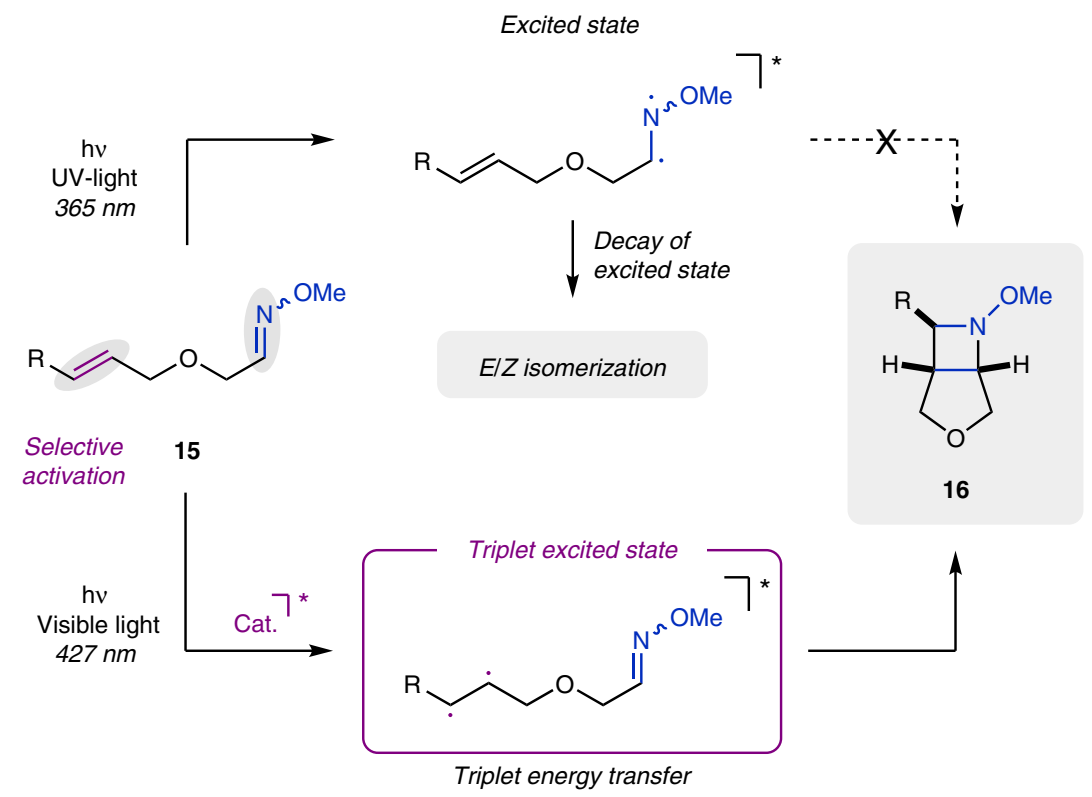

Fig. 2 Reaction design of the aza Paternò-Büchi reaction. Direct irradiation leads to unproductive excitation of the oxime moiety, while selective activation of the alkene via triplet energy transfer enables the $[2+2]$ photocycloaddition

\section{Table 1 Reaction optimization}

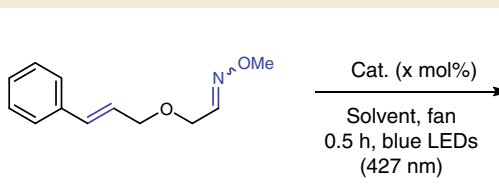

15

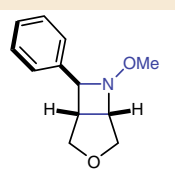

16 (>20:1 d.r.)

\begin{tabular}{|c|c|c|c|c|c|}
\hline Entry & Catalyst (mol\%) & Wavelength (nm) & Solvent & Concentration (M) & Yield (\%) \\
\hline $1^{b}$ & - & 365 & $\mathrm{CH}_{2} \mathrm{Cl}_{2}$ & 0.01 & 6 \\
\hline $2^{c}$ & Xanthone (30) & 365 & $\mathrm{MeCN}$ & 0.01 & 43 \\
\hline 3 & {$\left[\mathrm{Ru}(\mathrm{bpy})_{3}\right]\left(\mathrm{PF}_{6}\right)_{2}(2.5)$} & 427 & THF & 0.01 & - \\
\hline 4 & $\operatorname{Ir}(\mathrm{ppy})_{3}(2.5)$ & 427 & THF & 0.01 & 39 \\
\hline 5 & $17 \cdot \mathrm{PF}_{6}(2.5)$ & 427 & THF & 0.01 & 97 \\
\hline 6 & $17 \bullet P F_{6}(2.5)$ & 427 & $\mathrm{CH}_{2} \mathrm{Cl}_{2}$ & 0.025 & 72 \\
\hline 7 & $17 \bullet P F_{6}(2.5)$ & 427 & $\mathrm{MeOH}$ & 0.025 & 87 \\
\hline 8 & $17 \cdot P F_{6}(2.5)$ & 427 & EtOAc & 0.025 & 87 \\
\hline 9 & $17 \cdot \mathrm{PF}_{6}(2.5)$ & 427 & acetone & 0.025 & 86 \\
\hline 10 & $17 \bullet P F_{6}(2.5)$ & 427 & $\mathrm{MeCN}$ & 0.025 & 88 \\
\hline 11 & $17 \bullet P F_{6}(2.5)$ & 427 & THF & 0.025 & 93 \\
\hline 12 & $17 \bullet P F_{6}(2.5)$ & 427 & THF & 0.05 & 88 \\
\hline 13 & $17 \bullet P F_{6}(2.5)$ & 427 & $\mathrm{THF}$ & 0.10 & 90 \\
\hline 14 & $17 \bullet P F_{6}(1.0)$ & 427 & $\mathrm{THF}$ & 0.01 & 96 \\
\hline 15 & $17 \cdot P F_{6}(0.5)$ & 427 & THF & 0.01 & 98 \\
\hline 16 & - & 427 & $\mathrm{THF}$ & 0.01 & - \\
\hline $17^{d}$ & $17 \cdot P F_{6}(0.5)$ & - & THF & 0.01 & - \\
\hline
\end{tabular}

Conditions: Reactions performed on $0.1 \mathrm{mmol}$ scale under irradiation with a blue LED light ( $427 \mathrm{~nm}$ ) for $0.5 \mathrm{~h}$ at ambient temperature (fan cooling) aYield determined by quantitative ${ }^{1} \mathrm{H}$ NMR analysis from the crude mixture using an internal standard

bFor $24 \mathrm{~h}$

cFor $12 \mathrm{~h}$

dRun in the dark

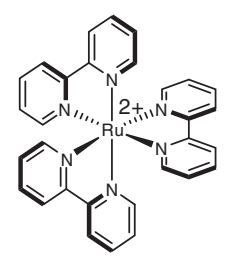

$\mathrm{Ru}(\mathrm{bpy})_{3}{ }^{2+}$

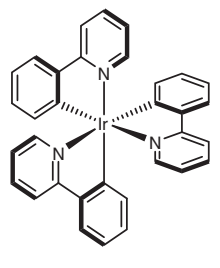

$\operatorname{Ir}(\text { ppy })_{3}$

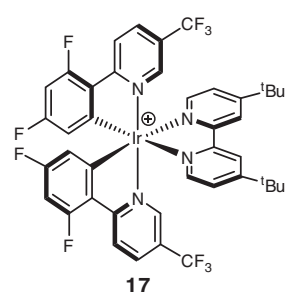

17 
Table 2 Evaluation of oxime and hydrazone substrates

a

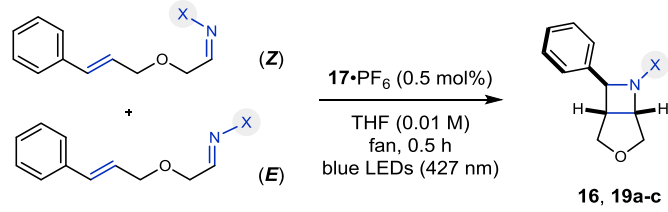

\begin{tabular}{|c|c|c|c|c|c|}
\hline Entry & Substrate & & $Z / E$ ratio & Yield & d.r. ${ }^{a}$ \\
\hline 1 & & 15 & $1: 1.3$ & $\begin{array}{l}96 \% \\
91 \%^{\mathrm{c}}\end{array}$ & $\begin{array}{l}>20: 1 \\
>20: 1\end{array}$ \\
\hline 2 & & $18 \mathrm{a}$ & $1: 1.3$ & $\begin{array}{l}96 \% \\
90 \%^{\mathrm{c}}\end{array}$ & $\begin{array}{l}16: 1 \\
20: 1\end{array}$ \\
\hline 3 & & $18 \mathrm{~b}$ & $1: 1.1$ & $54 \%$ & $>20: 1$ \\
\hline 4 & HBoc & $18 \mathrm{c}$ & $1: 2.6$ & $62 \%$ & $13: 1$ \\
\hline $5^{\mathrm{b}}$ & & 18d & $0: 1$ & $0 \%$ & - \\
\hline
\end{tabular}

Conditions: Reactions were performed with $0.25 \mathrm{mmol}$ substrate and $0.5 \mathrm{~mol} \% 17 \cdot \mathrm{PF}_{6}$ in $\mathrm{TH}$ $(0.01 \mathrm{M})$ at ambient temperature under blue LED irradiation $(427 \mathrm{~nm})$ for $0.5 \mathrm{~h}$ adiastereomeric ratio (d.r.) was determined by ${ }^{1} \mathrm{H}$ NMR from the crude reaction mixture run for $16 \mathrm{~h}$

b

creaction performed on gram scale

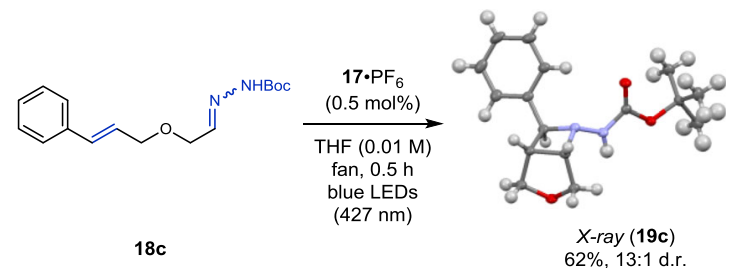

a Oximes and hydrazones in the aza Paternò-Büchi reaction. b crystal structure of azetidine 19c. d.r. $=$ diastereomeric ratio

was formed in 39\% yield (entry 4, Table 1). Reaction of oxime $\mathbf{1 5}$ with $2.5 \mathrm{~mol} \%$ of $\operatorname{Ir}\left[\mathrm{dF}\left(\mathrm{CF}_{3}\right) \text { ppy }\right]_{2}(\mathrm{dtbbpy}) \mathrm{PF}_{6}\left(\mathbf{1 7} \cdot \mathrm{PF}_{6}\right)$ resulted in the formation of azetidine $\mathbf{1 6}$ in increased yields of $97 \%$ and $>20: 1$ diastereomeric ratios (d.r.) (entry 5, Table 1). The potency of this photocatalyst was previously established by Yoon group $^{65,66}$ in the $[2+2]$ cycloaddition between alkenes for the formation of functionalized cyclobutanes. Further reaction optimization identified THF as optimal solvent resulting in $98 \%$ yield of azetidine 16 with catalyst loadings of only $0.5 \mathrm{~mol} \%$ 17. $\mathrm{PF}_{6}$ (entry 15 , Table 1 ). Finally, control reactions revealed that both light and photocatalyst were necessary for the $[2+2]$ photocycloaddition to proceed (entries 16-17, Table 1) ${ }^{67-71}$.

Substrate scope of aza Paternò-Büchi reaction. With optimized reaction conditions established, we next evaluated different oximes and hydrazones for their ability to undergo the $[2+2]$ cycloaddition (Table 2). It is important to note that all substrates were prepared and used as mixtures of $E / Z$ oxime or hydrazone isomers. However, the observed diastereoselectivity of the azetidine products formed was found to be independent of the $E / Z$ ratio of the substrate. O-Benzyl oxime 18 a converted smoothly to $19 \mathrm{a}$ in $96 \%$ yield and very good diastereoselectivity of $>20: 1$ d.r., providing similar results as the $O$-methyl oxime 15 (entries 1-2, Table 2a). Notably, the reaction can be performed on gram scale at slightly higher concentration with no significant decrease in yield. Free oxime $\mathbf{1 8 b}$ and $N$-Boc hydrazone $18 \mathrm{c}$ were reacted under the optimized conditions, and the corresponding azetidines 19b and 19c were isolated in 54 and 62\% yield, respectively (entries 3-4, Table 2a). Additionally, the structure of 19c was subsequently verified by X-ray analysis (Table $2 \mathrm{~b}$ ). Interestingly, no reaction was observed with $N, N$-dimethyl hydrazone $\mathbf{1 8 d}$ and only unreacted starting material was recovered from the reaction mixture (entry 5, Table 2a). While other substituted imines (e.g., $N$-tosyl imines) successfully underwent the aza Paternò-Büchi reaction, the poor stability towards hydrolysis of the corresponding substrates prohibited sufficient purification and led to reproducibility issues.

We subsequently investigated the scope of the aza Paternò-Büchi reaction with a particular emphasis on functional group tolerance and the ability to rapidly construct functionalized azetidines (Fig. 3). The optimized conditions proved efficient for a variety of electronically diverse styrenes including both electronrich and electron-deficient systems, affording the corresponding azetidines in excellent yields and diastereoselectivities (20-24). Furthermore, different substitution patterns on the styrene moiety as well as the substrate backbone including esters and sulfonamides were well tolerated, providing a set of densely substituted azetidines (25-29). While the developed transformation could be readily conducted under ambient atmosphere in short reaction times of $30 \mathrm{~min}$, substrates bearing increased steric constraints were found to require extended reaction times (3036). It was beneficial to conduct these transformations in degassed solvent to minimize undesired reactivity with atmospheric oxygen to ultimately obtain the desired azetidine products in high yields of up to $98 \%$. Specifically, oximes derived from methyl and phenyl ketones were converted to the corresponding azetidines $\mathbf{3 0}$ and $\mathbf{3 1}$ in excellent yield. The reaction is also amenable to heteroaromatic ketones-2-pyridyl azetidine $\mathbf{3 2}$ was afforded in $74 \%$ yield and excellent diastereoselectivity. Additionally, azetidine 33 bearing a lactone tether was formed in $60 \%$ (75\% brsm) after irradiation for $70 \mathrm{~h}$ at elevated temperatures. Importantly, substrates containing internal esters are often found less reactive in many ring-closing transformations, considering that the reaction can only proceed through the less favored $s$ trans ester conformation ${ }^{72}$. Furthermore, cyclic tetrasubstituted styrenes are compatible with the reaction conditions, providing tricyclic azetidine 34 in $98 \%$ yield. We also evaluated the feasibility of substrates to form azetidines fused to six-membered rings upon $[2+2]$ cycloaddition. The corresponding substrates were found to be significantly less reactive; nevertheless, chromane $\mathbf{3 5}$ and cyclohexane $\mathbf{3 6}$ were obtained after irradiation for $72 \mathrm{~h}$ in 42 and $93 \%$ yield, respectively. Based on the fact that many pharmaceutical or agrochemical products contain cyclic oxime or hydrazone motifs, we were interested in evaluating whether the aza Paternò-Büchi reaction protocol developed herein would allow for their late-stage modification. Cyclic oxime 38 derived from the herbicide safener isoxadifen ethyl (37) provided highly functionalized azetidine 39 in $87 \%$ yield, thus enabling late-stage modification of an industrially important oxime.

Subsequent investigations focused on the compatibility of dienes as alkene equivalent with the developed procedure for visible light-mediated aza Paternò-Büchi reactions (Fig. 4) ${ }^{66}$. After reacting the corresponding diene $\mathbf{4 0}$ under the optimized conditions, strained bicycle $\mathbf{4 1}$ was isolated as the product of the reaction in 39\% yield. Similarly, azetidine $\mathbf{4 3}$ was obtained in $99 \%$ yield. Notably, no products resulting from competing $[4+2]$ cycloadditions were observed in either one of these reactions.

Mechanistic investigations. Ensuing efforts focused on gaining additional insights into the controlling features of the visible 


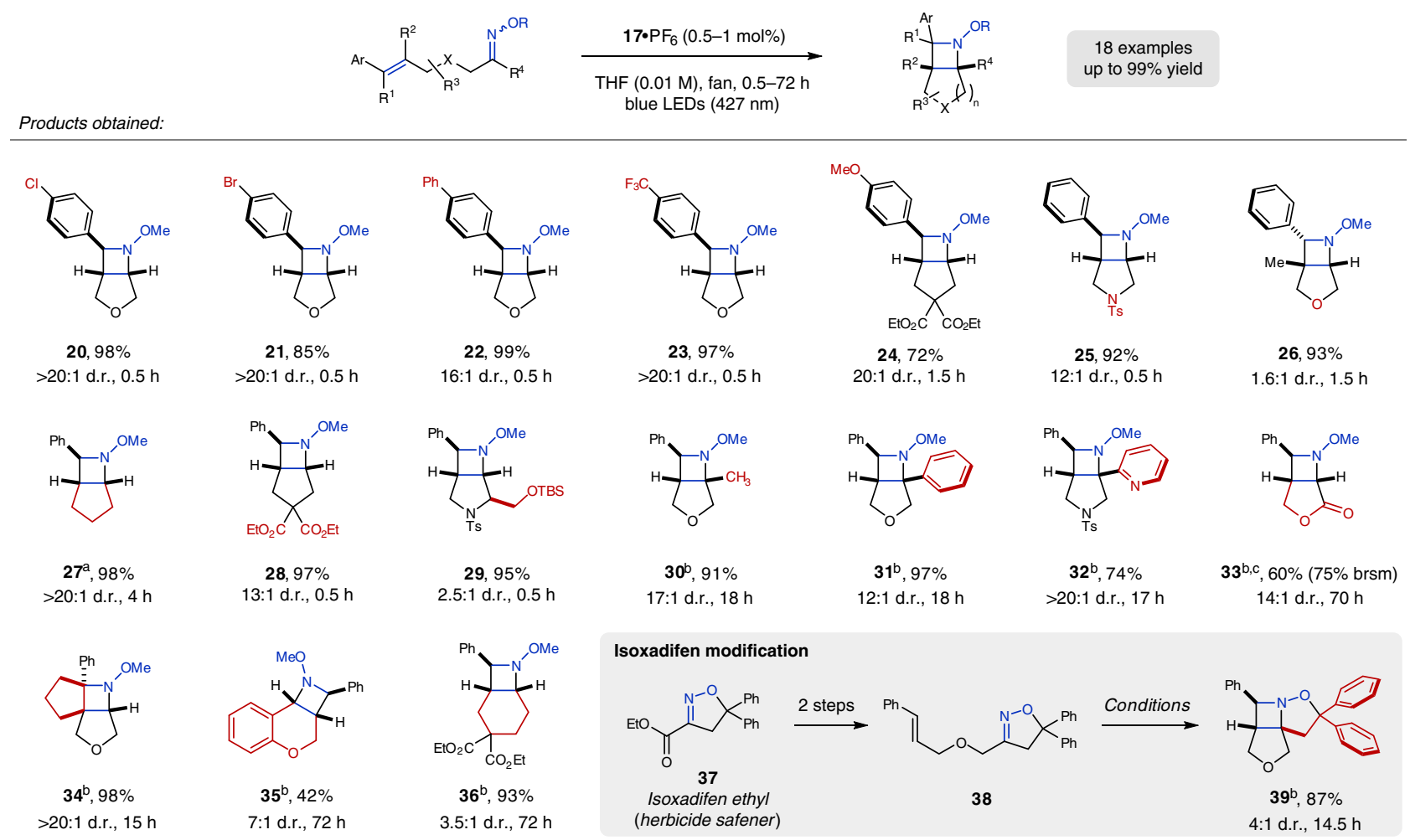

Fig. 3 Scope of the $[2+2]$ cycloaddition reaction. Reactions were performed with $0.25 \mathrm{mmol}$ substrate (mixture $E / Z$ oxime isomers) and $0.5-1.0$ mol\% 17•PF 6 in THF ( $0.01 \mathrm{M})$ at ambient temperature (fan cooling) under blue LED irradiation ( $427 \mathrm{~nm}$ ) unless noted; diastereomeric ratios (d.r.) were determined by ${ }^{1} \mathrm{H}$ NMR from the crude reaction mixture; isolated yields refer to the mixture of diastereomers (major diastereomer is given); ${ }^{a}$ on 500 mg scale; bunder $\mathrm{N}_{2}$ atmosphere; ${ }^{c}$ run at $82^{\circ} \mathrm{C}$

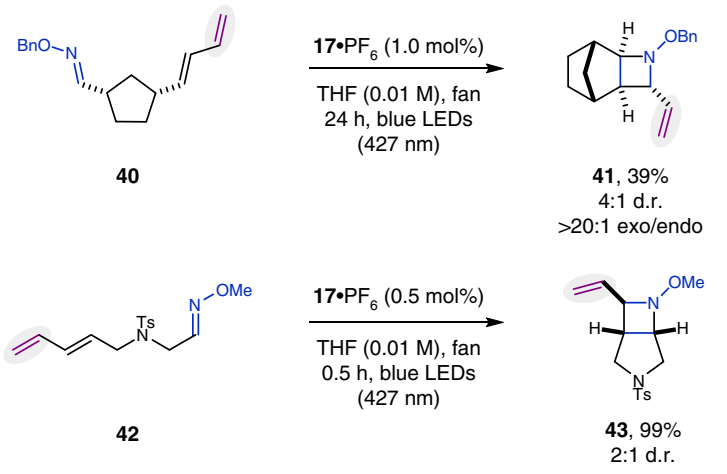

Fig. 4 Aza Paternò-Büchi reaction utilizing dienes. Reactions were performed with $0.25 \mathrm{mmol}$ substrate; diastereomeric ratios (d.r.) were determined by ${ }^{1} \mathrm{H}$ NMR from the crude reaction mixture; isolated yields refer to the mixture of diastereomers (major diastereomer is given)

light-mediated aza Paternò-Büchi reaction. A Stern-Volmer quenching study unambiguously showed that the styrene moiety is necessary for productive quenching of the photocatalyst, while the corresponding oxime $\mathbf{4 5}$ lacking a styrene moiety does not result in significant quenching of $\mathbf{1 7} \cdot \mathrm{PF}_{6}$ (Fig. 5a). We postulate that a photoredox process is unlikely under the optimized reaction conditions as the excited state redox potentials of $17 \cdot \mathrm{PF}_{6}$ $\left(E_{1 / 2}{ }^{\mathrm{III} / \mathrm{II}}=+1.21 \mathrm{~V} \text { vs. SCE; } E_{1 / 2}{ }^{\mathrm{IV} / \mathrm{III}}{ }^{\star}=-0.89 \mathrm{~V} \text { vs. SCE }\right)^{73}$ are not sufficient for an effective oxidation or reduction of substrate 15 (see Supplementary Methods for additional details). To further confirm this hypothesis, a series of control experiments was conducted, which imply that a triplet energy transfer mechanism from the photocatalyst to the styrene moiety of the substrate is operative. Styrenes possess a triplet energy $\left(E_{\mathrm{T}}\right)$ of $\sim 60 \mathrm{kcal} \mathrm{mol}^{-1}$, which suggest that $17 \cdot \mathrm{PF}_{6}\left(E_{\mathrm{T}}=62 \mathrm{kcal} \mathrm{mol}^{-1}\right)$ is capable of sensitizing substrate $15^{58}$. In comparison, the efficiency of this transformation significantly decreases with photocatalysts that have a triplet energy below $60 \mathrm{kcal} \mathrm{mol}^{-1}$. While $\mathrm{fac}$ - $\operatorname{Ir}(\mathrm{ppy})_{3}$ $\left(E_{\mathrm{T}}=58 \mathrm{kcal} \mathrm{mol}^{-1}\right)$ is still able to mediate product formation, albeit less efficiently, $\mathrm{Ru}(\mathrm{bpy})_{3}{ }^{2+}\left(E_{\mathrm{T}}=49 \mathrm{kcal} \mathrm{mol}^{-1}\right)$ was found incapable of catalyzing the desired aza Paternò-Büchi reaction (Fig. $5 \mathrm{~d})^{74}$. Consistent with this hypothesis, substrates bearing terminal alkenes with significantly higher triplet energy ( 76-84 $\left.\mathrm{kcal} \mathrm{mol}^{-1}\right)^{58}$ were not found to undergo the desired transformation, but instead resulted in exclusive isolation of starting material (see Supplementary Methods for additional details). Additionally, the aza Paternò-Büchi reaction developed herein was found to be stereoconvergent, as both (E)- and (Z)-15 gave identical results under standard conditions (Fig. 5b). The interconversion of both styrene isomers upon photosensitization is fast and occurs at a rate similar to product formation (see Supplementary Fig. 4). Interestingly, monitoring the oxime isomer ratio of $\mathbf{1 5}$ over the course of the reaction revealed that oxime $E / Z$ scrambling occurs at low conversion (Fig. 5c). In particular, within the first $2 \mathrm{~min}$ of the reaction an increase in ( $Z$ )-oxime concentration was observed along with a change in $E / Z$ ratio from 1.6:1 to $1: 1$. This observation cannot be accounted for based on the faster conversion of $(E)$-oxime to azetidine $\mathbf{1 6}$ in comparison to $(Z)$-oxime. In contrast, no change in $E / Z$ ratio was observed for compound 45 lacking the styrene moiety. Based on the results obtained in these investigations, we propose a reaction mechanism that relies on a styrene triplet manifold (intermediate I, Fig. 5). Efficient triplet energy transfer from photoexcited $\mathbf{1 7} \cdot \mathrm{PF}_{6}$ accessible upon irradiation with visible light results in a triplet styrene (intermediate I) that undergoes subsequent reversible 
a Stern-volmer quenching studies

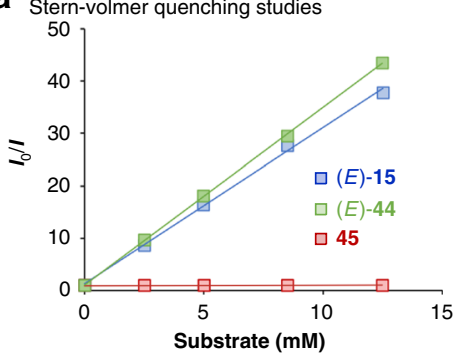

b Stereoconvergence of Aza Paternò-Büchi reaction

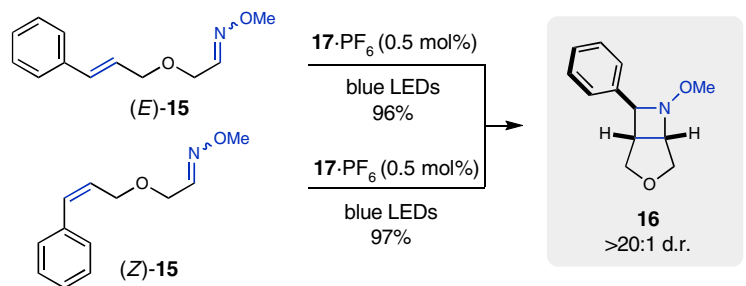

C Oxime $E / Z$ isomerization during course of reaction

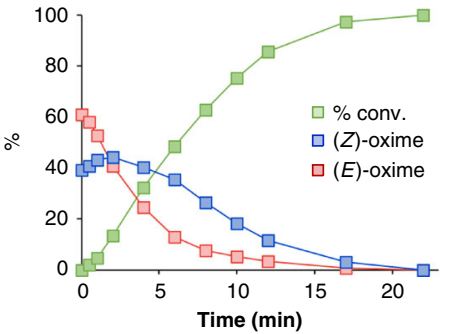<smiles>CON=CCOCC=Cc1ccccc1</smiles>

$(E)-15$<smiles>CCOC/C=C/c1ccccc1</smiles>

(E)-44<smiles>CON=CCOCCCc1ccccc1</smiles>

45
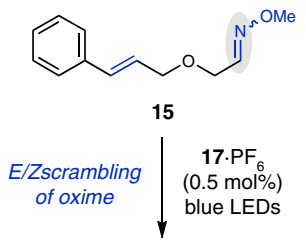

16

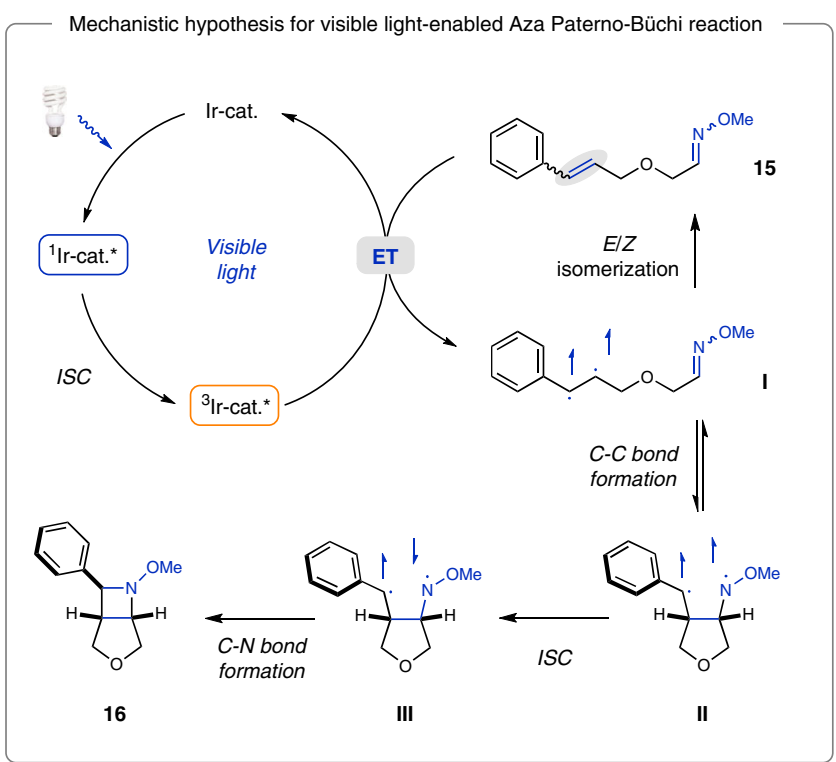

d Correlation of triplet energy and reaction efficiency

$\begin{array}{cccc}\text { Entry } & \text { Photocatalyst }(2.5 \mathrm{~mol} \%) & E_{\mathrm{T}}\left(\mathrm{kcal} \mathrm{mol}^{-1}\right) & \text { Yield } \\ 1 & 17 \cdot \mathrm{PF}_{6} & 62 & 97 \% \\ 2 & \mathrm{fac}-\mathrm{Ir}(\mathrm{ppy})_{3} & 58 & 39 \% \\ 3 & {\left[\mathrm{Ru}(\mathrm{bpy})_{3}\right]\left(\mathrm{PF}_{6}\right)_{2}} & 49 & 0 \%\end{array}$

Fig. 5 Mechanistic investigations of the title reaction. a Stern-Volmer quenching studies. b Stereoconvergence of the aza Paternò-Büchi reaction. c Oxime $E / Z$ isomerization during the course of the reaction. $\mathbf{d}$ Correlation of triplet energy and reaction efficiency (see Supplementary Methods for details). $E_{\mathrm{T}}=$ triplet energy; $\mathrm{ET}=$ energy transfer; ISC = intersystem crossing

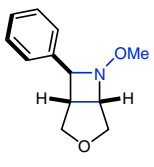

16

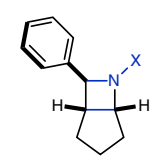

$27(\mathrm{X}=\mathrm{OMe})$ $\mathrm{Zn} / \mathrm{HCl}$ (aq.)

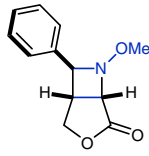

33

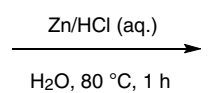

$\mathrm{H}_{2} \mathrm{O}, 80^{\circ} \mathrm{C}, 1 \mathrm{~h}$

$$
\begin{aligned}
& \mathrm{RuCl}_{3} \cdot \times \mathrm{H}_{2} \mathrm{O} \\
& \mathrm{H}_{5} \mathrm{IO}_{6} \text { (30 equiv.) } \\
& \mathrm{H}_{2} \mathrm{O} / \mathrm{MeCN} / \mathrm{CCl}_{4}
\end{aligned}
$$

$\mathrm{Zn} / \mathrm{HCl}($ aq.)

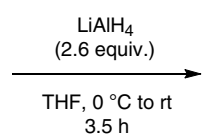

3.5
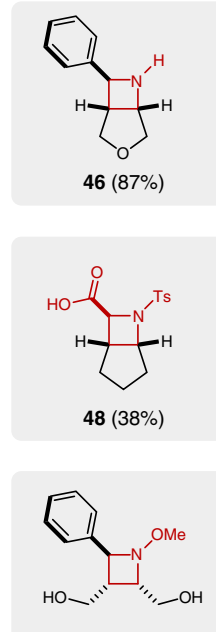

$49(84 \%)$
Fig. 6 Synthetic modifications of azetidine products. The accessed azetidines can be converted to the corresponding unprotected azetidine (46), azetidine-2-carboxylic acid (48), or monocyclic azetidine (49)

$C-C$ bond formation to result in a 1,4-biradical (intermediate II). This intermediate allows for free rotation around the $C-N$ bond that ultimately leads to the observed $E / Z$ scrambling of the oxime after ring-opening (intermediate I) and relaxation back to the ground state. Alternatively, intermediate II can undergo intersystem crossing (ISC) (intermediate III), and deliver the azetidine product (16) after the final $C-N$ bond-forming step. Importantly, the biradical nature of the involved triplet intermediates leads to complete loss of stereoinformation, which results in the formation of the same diastereomer independent of the oxime or alkene isomer ratios of the starting material.

Synthetic applications. The developed [2+2] cycloaddition protocol enables rapid access to highly functionalized azetidines under mild conditions that can function as versatile building blocks to undergo further diversification (Fig. 6). The azetidine $\mathrm{N}-\mathrm{O}$ bond can be readily cleaved with zinc metal under acidic conditions providing free azetidine 46 in $87 \%$ yield. To demonstrate the utility of the 2-phenylazetidine motif accessible by this method, azetidine 27 was converted to the corresponding $N$-Ts azetidine 47 (70\% yield over 2 steps), which is amenable to phenyl ring oxidation utilizing $\mathrm{RuCl}_{3} / \mathrm{H}_{5} \mathrm{IO}_{6}$ to provide carboxylic acid 48 in 38\% yield. Notably, 48 represents a previously inaccessible analog of the non-proteinogenic amino acid azetidine-2carboxylic acid (Aze). Finally, the lactone tether of azetidine 33 is readily cleaved with $\mathrm{LiAlH}_{4}$ to provide 49 in $84 \%$ yield, resembling the product of a net intermolecular [2+2] cycloaddition reaction.

\section{Discussion}

We herein report the development of a visible light-mediated aza Paternò-Büchi reaction between alkene and oxime moieties that results in the direct formation of functionalized azetidines, with yields of up to $99 \%$ and $>20: 1$ d.r. The approach described 
relies on the selective activation of the alkene functionality upon energy transfer from a suitable photocatalyst to its corresponding triplet state. As a result, the aza Paternò-Büchi reaction developed herein overcomes previous challenges associated with the excitation of functionalized imines and resulting undesired competing reaction paths. We expect that this strategy will provide a new platform for the facile synthesis of azetidines and will enable further advancements in developing new enabling $[2+2]$ cycloadditions involving carbon-nitrogen double bonds.

\section{Methods}

Representative procedure. A test tube was charged with $15(0.25 \mathrm{mmol}), \mathbf{1 7} \cdot \mathrm{PF}_{6}$ $(0.5 \mathrm{~mol} \%)$, and tetrahydrofuran $(25 \mathrm{~mL})$, sealed with a rubber septum and placed in front of a $40 \mathrm{~W}$ PR160-427 nm Kessil light ( $5 \mathrm{~cm}$ distance; $100 \%$ intensity). Upon completion as judged by thin layer chromatography analysis, the reaction mixture was concentrated in vacuum. The diastereomeric ratio was determined by ${ }^{1} \mathrm{H}$ NMR (proton nuclear magnetic resonance) analysis from the crude reaction mixture, before purification by flash column chromatography (10-20\% EtOAc/ hexanes) to afford pure $\mathbf{1 6}$.

\section{Data availability}

Experimental data as well as ${ }^{1} \mathrm{H}$ and ${ }^{13} \mathrm{C}$ NMR spectra for all new compounds prepared in the course of these studies are provided in the supplementary information of this manuscript. The X-ray crystallographic coordinates for compound 19c have been deposited at the Cambridge Crystallographic Data Center (CCDC) with the accession code 1873931 (https://doi.org/10.5517/ccdc.csd.cc20wzd9). These data can be obtained free of charge from The Cambridge Crystallographic Data Center via www.ccdc.cam.ac. uk/data_request/cif. All other data including synthetic procedures are available in the supplementary information files.

Received: 22 July 2019; Accepted: 17 October 2019; Published online: 08 November 2019

\section{References}

1. Vitaku, E., Smith, D. T. \& Njardarson, J. T. Analysis of the structural diversity, substitution patterns, and frequency of nitrogen heterocycles among U.S. FDA approved pharmaceuticals. J. Med. Chem. 57, 10257-10274 (2014).

2. Kerns, E. H. \& Di, L. Drug-Like Properties: Concepts, Structure Design and Methods 1st edn 137-168 (Academic Press, 2008).

3. St. Jean, D. J. \& Fotsch, C. Mitigating heterocycle metabolism in drug discovery. J. Med. Chem. 55, 6002-6020 (2012).

4. Shu, Y.-Z., Johnson, B. M. \& Yang, T. J. Role of biotransformation studies in minimizing metabolism-related liabilities in drug discovery. AAPS J. 10, 178-192 (2008).

5. Lovering, F., Bikker, J. \& Humblet, C. Escape from Flatland: increasing saturation as an approach to improving clinical success. J. Med. Chem. 52, 6752-6756 (2009).

6. Lovering, F. Escape from Flatland 2: complexity and promiscuity. Med. Chem. Commun. 4, 515-519 (2013).

7. Antermite, D., Degennaro, L. \& Luisi, R. Recent advances in the chemistry of metallated azetidines. Org. Biomol. Chem. 15, 34-50 (2017).

8. Brandi, A., Cicchi, S. \& Cordero, F. M. Novel syntheses of azetidines and azetidinones. Chem. Rev. 108, 3988-4035 (2008).

9. Cromwell, N. H. \& Phillips, B. The azetidines. Recent Synth. Dev. Chem. Rev. 79, 331-358 (1979)

10. Yoda, H., Takahashi, M. \& Sengoku, T. in Heterocycles in Natural Product Synthesis 1 st edn (eds Majumdar, K. C. \& Chattopadhyay, S. K.) (Wiley-VCH, 2011).

11. Clayden, C., Greeves, N. \& Warren, S. Organic Chemistry 2nd edn 805-807 (Oxford Univ. Press, 2012)

12. Warren, S. \& Wyatt, P. Organic Synthesis: The Disconnection Approach 2nd edn 217-219 (Wiley, 2008)

13. Funke, W. Synthesis and properties of 1-azabicyclo[1.1.0]butanes. Angew. Chem. Int. Ed. 8, 70-71 (1969).

14. Funke., W. Über synthesen und reaktionen von 1-aza-bicyclo[1.1.0]butanen. Chem. Ber. 102, 3148-3159 (1969).

15. Gianatassio, R. et al. Strain-release amination. Science 351, 241-246 (2016).

16. Lopchuk, J. M. et al. Strain-release heteroatom functionalization: development, scope, and stereospecificity. J. Am. Chem. Soc. 139, 3209-3226 (2017).

17. Gianatassio, R. \& Kadish, D. Direct alkylation of 1-azabicyclo[1.1.0]butanes. Org. Lett. 21, 2060-2063 (2019).
18. Fawcett, A., Murtaza, A., Gregson, C. H. U. \& Aggarwal, V. K. Strain-releasedriven homologation of boronic esters: application to the modular synthesis of azetidines. J. Am. Chem. Soc. 141, 4573-4578 (2019).

19. Pitts, C. R. \& Lectka, T. Chemical synthesis of $\beta$-lactams: asymmetric catalysis and other recent advances. Chem. Rev. 114, 7930-7953 (2014).

20. Alcaide, B., Almendros, P. \& Aragoncillo, C. $\beta$-Lactams: versatile building blocks for the stereoselective synthesis of non- $\beta$-lactam products. Chem. Rev. 107, 4437-4492 (2007).

21. Flores, D. M. \& Schmidt, V. A. Intermolecular $2+2$ carbonyl-olefin photocycloadditions enabled by $\mathrm{Cu}(\mathrm{I})$-Norbornene MLCT. J. Am. Chem. Soc. 141, 8741-8745 (2019).

22. Padwa, A. Photochemistry of the carbon-nitrogen double bond. Chem. Rev. 77, 37-68 (1977).

23. Pratt, A. C. The photochemistry of imines. Chem. Soc. Rev. 6, 63-81 (1977)

24. Stoermer, R. Über die Umlagerung der stabilen stereoisomeren in labile durch ultraviolettes Licht (TOGGLE). Ber. Dtsch. Chem. Ges. 44, 637-668 (1911).

25. Amin, J. H. \& de Mayo, P. The irradiation of aryl aldoximes. Tetrahedron Lett. 4, 1585-1589 (1963).

26. Anderson, D. G. \& Wettermark, G. Photoinduced isomerizations in anils. J. Am. Chem. Soc. 87, 1433-1438 (1965).

27. Stenberg, V. I., Barks, P. A., Bays, D., Hammargren, D. D. \& Rao, D. V. Nitrogen photochemistry. Syn and anti isomers of semicarbazones. J. Org. Chem. 33, 4402-4406 (1968).

28. Padwa, A. \& Albrecht, F. Photoisomerization about the carbon-nitrogen double bond of an oxime ether. J. Am. Chem. Soc. 94, 1000-1002 (1972).

29. Rainoldi, G., Faltracco, M., Lo Presti, L., Silvani, A. \& Lesma, G. Highly diastereoselective entry into chiral spirooxindole-based 4-methyleneazetidines via formal $[2+2]$ annulation reaction. Chem. Commun. 52, 11575-11578 (2016).

30. Nakamura, I., Nemoto, T., Yamamoto, Y. \& de Meijere, A. Thermally induced and silver-salt-catalyzed $[2+2]$ cycloadditions of imines to (alkoxymethylene) cyclopropanes. Angew. Chem. Int. Ed. 45, 5176-5179 (2006).

31. Akiyama, T., Daidouji, K. \& Fuchibe, K. Cu(I)-catalyzed enantioselective [2 +2 ] cycloaddition of 1-methoxyallenylsilane with $\alpha$-imino ester: chiral synthesis of $\alpha, \beta$-unsaturated acylsilanes. Org. Lett. 5, 3691-3693 (2003).

32. Uyehara, T. et al. Lewis acid-promoted $[2+2]$ azetidine annulation of $N$ acylaldimines with allyltriisopropylsilane. Chem. Lett. 24, 789-790 (1995).

33. Cabral, J., Laszlo, P. \& Montaufier, M. T. Schizoid reactivity of $N$-benzylidene aniline toward clay-catalyzed cycloadditions. Tetrahedron Lett. 29, 547-550 (1988).

34. Aben, R. W. M., Smit, R. \& Scheeren, J. W. High-pressure-promoted [2+2] cycloadditions of imines with electron-rich alkenes. A simple route to azetidines and $\beta$-amino carbonyl compounds. J. Org. Chem. 52, 365-370 (1987).

35. Behnke, N. E., Lovato, K., Yousufuddin, M. \& Kürti, L. Titanium-mediated synthesis of spirocyclic $\mathrm{NH}$-azetidines from oxime ethers. Angew. Chem. Int. Ed. 58, 1-6 (2019).

36. Anderson, L. L. Applications of nitrones for the synthesis of heterocyclic compounds. Asian J. Org. Chem. 5, 9-30 (2019).

37. Son, J., Reidl, T. W., Kim, K. H., Wink, D. J. \& Anderson, L. L. Generation and rearrangement of N,O-dialkenylhydroxylamines for the synthesis of 2aminotetrahydrofurans. Angew. Chem. Int. Ed. 57, 6597-6600 (2018).

38. Kroc, M. A., Prajapati, A., Wink, D. J. \& Anderson, L. L. Cascade synthesis of 3 -functionalized indoles from nitrones and their conversion to cycloheptanone-fused indoles. J. Org. Chem. 83, 1085-1094 (2018).

39. Reidl, T. W., Son, J., Wink, D. J. \& Anderson, L. L. Facile synthesis of azetidine nitrones and diastereoselective conversion into densely substituted azetidines. Angew. Chem. Int. Ed. 56, 11579-11583 (2017)

40. Dowling, M. S., Fernando, D. P., Hou, J., Liu, B. \& Smith, A. C. Two scalable syntheses of (S)-2-methylazetidine. J. Org. Chem. 81, 3031-3036 (2016).

41. Fritz, S. P., Moya, J. F., Unthank, M. G., McGarrigle, E. M. \& Aggarwal, V. K. An efficient synthesis of azetidines with (2-bromoethyl)sulfonium triflate. Synthesis 44, 1584-1590 (2012).

42. Andresini, M. et al. Azetidine-borane complexes: synthesis, reactivity, and stereoselective functionalization. J. Org. Chem. 83, 10221-10230 (2018).

43. Fischer, G., Fritz, H. \& Prinzbach, H. An intramolecular imine/ene-photo-[2 +2]-cycloaddition reaction. Tetrahedron Lett. 27, 1269-1272 (1968).

44. Marterer, W. et al. The azo(Azoxy) functionality as a $\pi_{2}$ component in photo $[2+2]$ cycloadditions 'syn'- and 'anti'-3,4-diazatricyclo[4.2.2.2 2,5]dodeca-3,7diene, syntheses, photolyses, X-ray-structure analysis, and PE spectra. Helv. Chim. Acta 71, 1937-1965 (1988).

45. Fischer, G. et al. Proximate, syn-periplanar, rigid imine(nitrone)/Ene-, and diazene(diazeneoxy)/Ene systems: syntheses, homoconjugate reactivity and photochemistry. Eur. J. Org. Chem. 2000, 743-762 (2000).

46. Howard, K. A. \& Koch, T. H. Photochemical reactivity of keto imino ethers. V. $(2+2)$ Photocycloaddition to the carbon-nitrogen double bond of 3ethoxyisoindolone. J. Am. Chem. Soc. 97, 7288-7298 (1975). 
47. Swenton, J. S. \& Hyatt, J. A. Photosensitized cycloadditions to 1,3-dimethyl-6azauracil and 1,3-dimethyl-6-azathymine. Imine linkage unusually reactive toward photocycloaddition. J. Am. Chem. Soc. 96, 4879-4885 (1974).

48. Nishio, T. \& Omote, Y. Photocycloaddition of quinoxalin-2-ones and benzoxazin-2-ones to aryl alkenes. J. Chem. Soc. Perk. Trans. 1, 2611-2615 (1987).

49. Nishio, T. The $(2+2)$ photocycloaddition of the carbon-nitrogen double bond of quinoxalin-2 $\left({ }^{1} \mathrm{H}\right)$-ones to electron-deficient olefins. J. Org. Chem. 49, 827-832 (1984).

50. Sakamoto, R., Inada, T., Sakurai, S. \& Maruoka, K. [2+2] Photocycloadditions between the carbon-nitrogen double bonds of imines and carbon-carbon double bonds. Org. Lett. 18, 6252-6255 (2016).

51. Kumarasamy, E., Kandappa, S. K., Raghunathan, R., Jockusch, S. \& Sivaguru, J. Realizing an aza Paternò-Büchi reaction. Angew. Chem. Int. Ed. 56, 7056-7061 (2017).

52. Boyington, A. J., Seath, C. P., Zearfoss, A. M., Xu, Z. \& Jui, N. T. Catalytic strategy for the regioselective arylethylamine synthesis. J. Am. Chem. Soc. 141, 4147-4153 (2019).

53. Boyington, A. J., Riu, M.-L. \& Jui, N. T. Anti-Markovnikov hydroarylation of unactivated olefins using pyridyl radical intermediates. J. Am. Chem. Soc. 139, 6582-6585 (2017).

54. Zhao, K. et al. Catalytic ring expansions of cyclic alcohols enabled by protoncoupled electron transfer. J. Am. Chem. Soc. 141, 8752-8757 (2019).

55. Nguyen, S. T., Zhu, Q. \& Knowles, R. R. PCET-enabled olefin hydroamination reactions with $N$-alkyl amides. ACS Catal. 9, 4502-4507 (2019).

56. Kottisch, V., Suppen, M. J. \& Fors, B. P. Enhancing temporal control and enabling chain-end modification in photoregulated cationic polymerizations by using iridium-based catalysts. Angew. Chem. Int. Ed. 57, 8260-8264 (2018).

57. Kottisch, V., Michaudel, Q. \& Fors, B. P. Photocontrolled Interconversion of cationic and radical polymerizations. J. Am. Chem. Soc. 139, 10665-10668 (2017).

58. Ni, T., Caldwell, R. A. \& Melton, L. A. The relaxed and spectroscopic energies of olefin triplets. J. Am. Chem. Soc. 111, 457-464 (1989).

59. Padwa, A., Bergmark, W. \& Pashayan, D. Mechanism of the photoreduction of $\mathrm{N}$-alkylbenzylidenimines. J. Am. Chem. Soc. 91, 2653-2660 (1969).

60. Padwa, A. \& Koehn, W. P. Photochemical reduction in the $N$-acylketimine system. J. Org. Chem. 40, 1896-1902 (1975).

61. Ohta, H. \& Tokumaru, K. Photochemistry of $\mathrm{N}$-(4-dimethylaminobenzylidene) aniline. Bull. Chem. Soc. Jpn. 48, 1669-1670 (1975).

62. Yoshida, M., Sakuragi, H., Nishimura, T., Ishikawa, S. \& Tokumaru, K. Nature of the excited triplet states in the photolysis of $O$-acyloximes. Chem. Lett. 4 , 1125-1130 (1975).

63. Roth, H. D. in PATAI's Chemistry of Functional Groups (ed. Marek, I.) (Wiley, 2010).

64. Romero, N. A. \& Nicewicz, D. A. Organic photoredox catalysis. Chem. Rev 116, 10075-10166 (2016).

65. Lu, Z. \& Yoon, T. P. Visible light photocatalysis of [2+2] styrene cycloadditions by energy transfer. Angew. Chem. Int. Ed. 51, 10329-10332 (2012).

66. Hurtley, A. E., Lu, Z. \& Yoon, T. P. [2+2] Cycloaddition of 1,3-dienes by visible light photocatalysis. Angew. Chem. Int. Ed. 53, 8991-8994 (2014).

67. Zhou, Q.-Q., Zou, Y.-Q., Lu, L.-Q. \& Xiao, W.-J. Visible-light-induced organic photochemical reactions through energy-transfer pathways. Angew. Chem. Int. Ed. 58, 1586-1604 (2019).

68. Zou, Y.-Q. et al. Visible light induced intermolecular [2+2]-cycloaddition reactions of 3-ylideneoxindoles through energy transfer pathway. Tetrahedron 68, 6914-6919 (2012).

69. Alonso, R. \& Bach, T. A chiral thioxanthone as an organocatalyst for enantioselective $[2+2]$ photocycloaddition reactions induced by visible light. Angew. Chem. Int. Ed. 53, 4368-4371 (2014).
70. Huang, X. et al. Direct visible-light-excited asymmetric Lewis acid catalysis of intermolecular [2+2] photocycloadditions. J. Am. Chem. Soc. 139, 9120-9123 (2017).

71. Zhu, M., Zheng, C., Zhang, X. \& You, S.-L. Synthesis of cyclobutane-fused angular tetracyclic spiroindolines via visible-light-promoted intramolecular dearomatization of indole derivatives. J. Am. Chem. Soc. 141, 2636-2644 (2019).

72. Pawar, D. W. et al. E and $\mathrm{Z}$ conformations of esters, thiol esters, and amides. J. Am. Chem. Soc. 120, 2108-2112 (1998)

73. Prier, C. K., Rankic, D. A. \& MacMillan, D. W. C. Visible light photoredox catalysis with transition metal complexes: applications in organic synthesis. Chem. Rev. 113, 5322-5363 (2013).

74. Strieth-Kalthoff, F., James, M. J., Teders, M., Pitzer, L. \& Glorius, F. Energy transfer catalysis mediated by visible light: principles, applications, directions. Chem. Soc. Rev. 47, 7190-7202 (2018).

\section{Acknowledgements}

We thank Dr. Jeff W. Kampf for X-ray crystallographic studies. C.S.S. thanks the Alfred P. Sloan Foundation, the David and Lucile Packard Foundation, and the Camille and Henry Dreyfus Foundation for fellowships. M.R.B. is grateful for a Peter A.S. Smith Endowment Award for Research.

\section{Author contributions}

M.R.B., A.D.R., and C.S.S. designed the experiments. M.R.B. and A.D.R. conducted and analyzed the experiments described in this report. M.R.B., A.D.R., and C.S.S. prepared this manuscript for publication.

\section{Competing interests}

The authors declare no competing interests.

\section{Additional information}

Supplementary information is available for this paper at https://doi.org/10.1038/s41467019-13072-x.

Correspondence and requests for materials should be addressed to C.S.S.

Peer review information Nature Communications thanks the anonymous reviewer(s) for their contribution to the peer review of this work.

Reprints and permission information is available at http://www.nature.com/reprints

Publisher's note Springer Nature remains neutral with regard to jurisdictional claims in published maps and institutional affiliations.

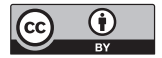

Open Access This article is licensed under a Creative Commons Attribution 4.0 International License, which permits use, sharing, adaptation, distribution and reproduction in any medium or format, as long as you give appropriate credit to the original author(s) and the source, provide a link to the Creative Commons license, and indicate if changes were made. The images or other third party material in this article are included in the article's Creative Commons license, unless indicated otherwise in a credit line to the material. If material is not included in the article's Creative Commons license and your intended use is not permitted by statutory regulation or exceeds the permitted use, you will need to obtain permission directly from the copyright holder. To view a copy of this license, visit http://creativecommons.org/ licenses/by/4.0/

(c) The Author(s) 2019 